\title{
Medición del módulo de Young en metales mediante la técnica de excitación por impulso
}

\author{
Young's modulus measurements in metals \\ using impulse excitation technique
}

\author{
Sebastián Tognana ${ }^{1}$, Susana Montecinos ${ }^{2}$, Walter Salgueiro ${ }^{1}$
}

\begin{abstract}
${ }^{1}$ IFIMAT, CIFICEN-CONICET-CICPBA-UNCPBA, Facultad de Ciencias Exactas, Universidad Nacional del Centro de la Provincia de Buenos Aires, Pinto 399, 7000 Tandil, Argentina y Comisión de Investigaciones Científicas de la Provincia de Bs. As., Calle 526 entre 10 y 11, 1900, La Plata, Buenos Aires, Argentina.

e-mail: stognana@exa.unicen.edu.ar; wsalgue@exa.unicen.edu.ar

${ }^{2}$ IFIMAT, Facultad de Ciencias Exactas, Universidad Nacional del Centro de la Provincia de Buenos Aires, Pinto 399, 7000 Tandil, Argentina, y Consejo Nacional de Investigaciones Científicas y Técnicas CONICET, Av. Rivadavia 1917, C1033AAJ, Buenos Aires, Buenos Aires, Argentina.

e-mail: dmonteci@exa.unicen.edu.ar
\end{abstract}

\section{RESUMEN}

La excitación por impulso como técnica no destructiva para el estudio de materiales permite determinar las constantes elásticas de los mismos por intermedio de la medición de la frecuencia natural de vibración de barras en diferentes modos y excitadas mediante un impacto puntual. En este trabajo se determinó el módulo de Young en muestras de cobre, aluminio y aleaciones de base cobre. Las mediciones se realizaron usando distintos modos de vibración: flexión en una barra apoyada y longitudinal. Como primer paso se determinó el módulo de Young de una muestra usando diferentes modos, encontrando consistencia en los resultados. Por otro lado, se determinó la variación del módulo de Young en función de la temperatura y parámetros microestructurales como el tamaño de grano. Se analizan las capacidades y desventajas del uso de la técnica de excitación por impulso como una alternativa de bajo costo en el ámbito de la investigación en metales y aleaciones.

Palabras clave: Técnica de Excitación por Impulso, Módulo de Young, aleaciones base cobre, tamaño de grano.

\section{ABSTRACT}

The impulse excitation, as non-destructive technique, allows to determine the elastic constants of materials by means of measurement of natural frequency of vibration of bars in different modes, excited by an impact in a point. In this work, the Young's modulus of copper, aluminum and copper-based alloys samples was determined. The measurements were performed using different vibration modes: flexural in supported bars, and longitudinal. As a first step, the Young's modulus of a sample was determined using different modes, finding consistency in results. On the other hand, the variation of the Young's modulus was determined as a function of the temperature and microstructural parameters such as grain size. The capabilities and disadvantages of using the impulse excitation technique as a low-cost alternative in the field of research in metals and alloys are analyzed.

Keywords: Impulse Excitation Technique, Young's modulus, copper-based alloys, grain size.

\section{INTRODUCCIÓN}

Numerosas técnicas son utilizadas para caracterizar y estudiar las propiedades mecánicas de materiales, además de su aplicación en el control de calidad en los procesos de producción. Entre los parámetros más importantes que caracterizan las propiedades mecánicas de los materiales se encuentra el módulo de Young, E. Éste 
resulta muy útil para el estudio de la respuesta mecánica, pero además es un parámetro fundamental para realizar cálculos y simulaciones vinculados a la estabilidad del material, produciendo resultados que son aplicados luego para analizar su comportamiento en condiciones de servicio. La medición de este parámetro se puede realizar por diferentes métodos que pueden ser estáticos o dinámicos. En particular la técnica de excitación por impulso (Impulse Excitation Technique, IET) exhibe como ventaja su condición de ser no destructiva, además de su rapidez de ejecución, fácil implementación y bajo costo. Además algunos autores le han atribuido una mayor precisión respecto a las técnicas estáticas convencionales [1]. Si bien no es tan utilizada como otras técnicas tradicionales aplicadas al estudio de las propiedades mecánicas, se reportan diferentes estudios en la bibliografía [2-4]. La técnica consiste en excitar las frecuencias naturales de una muestra por medio de un impacto puntual en una de sus caras. Generalmente se reportan estudios con muestras en forma de barras de sección rectangular excitando la vibración en modo flexión, pero pueden utilizarse barras de sección circular en modo flexión, y también en modo longitudinal.

Se encuentran en la literatura algunos estudios donde se reporta el módulo $E$ en distintas aleaciones base cobre, específicamente en aleaciones CuAlBe [5-8] y CuBe [9]. Sin embargo, la mayoría de ellos han sido determinados mediante técnicas destructivas. Para aplicaciones tales como sensores, resulta de gran importancia conocer la respuesta mecánica ante la variación de parámetros microestructurales y condiciones de ensayo. En particular, el tamaño de grano es un parámetro con fuerte influencia sobre las propiedades mecánicas. Por ejemplo, se ha reportado en aleaciones con memoria de forma CuAlBe que para mayores tamaños de grano el módulo $E$ y la tensión de inicio de la transformación martensítica disminuyen [3,10], mientras que al aumentar la temperatura de ensayo la tensión de inicio de la transformación martensítica aumenta [10].

Por otro lado, Miki et al. [11] estudió la influencia del tamaño de grano sobre la dureza en aleaciones $\mathrm{CuBe}$, encontrando que al aumentar el tamaño de grano se obtienen mayores valores de dureza, siendo este efecto más importante durante las primeras etapas de termo-endurecimiento.

En este trabajo se aplica la técnica IET específicamente para estudiar el módulo $E$ en muestras de cobre, aluminio y base de cobre. Asimismo, se determinó la variación del módulo de Young en función de la temperatura y tamaño de grano, el cual fue modificado mediante tratamientos térmicos específicos.

\section{MATERIALES Y MÉTODOS}

Mediante el dispositivo IET se estudiaron muestras de Al puro (99.8\% de pureza), cobre y aleaciones CuAl$\mathrm{Be}$ y $\mathrm{CuBe}$. Las aleaciones utilizadas en este trabajo fueron obtenidas comercialmente. El cobre corresponde a Cu electrolítico y fue obtenido como barras cilíndricas de $6.42 \mathrm{~mm}$ de diámetro y placas de $2.12 \mathrm{~mm}$ de espesor (Cu_placa). El aluminio fue medido en forma de barra de $12.7 \mathrm{~mm}$ x $12.5 \mathrm{~mm}$ x $87.6 \mathrm{~mm}$ mecanizada en fresa. La aleación $\mathrm{CuBe}$, de composición $\mathrm{Cu}-1.91 \mathrm{Be}-0.3(\mathrm{Ni}+\mathrm{Co}$ ) (\%p.) (con impurezas de $\mathrm{Si}, \mathrm{Fe}, \mathrm{Al}$ y $\mathrm{Pb}$ con contenidos $\leq 0.03 \% \mathrm{p}$.), fue obtenida en Roberto Cordes S.A. bajo la forma de barras cilíndricas de 6 $\mathrm{mm}$ de diámetro. La aleación CuAlBe, de composición Cu-11.41Al-0.50Be (A1) y Cu-11.40Al-0.55Be (A2) (\%p.), fue obtenida de Trefimetaux S.A. bajo la forma de barras cilíndricas de 3.5 y $15 \mathrm{~mm}$ de diámetro, para A1 y A2, respectivamente. Los tamaños de grano en las muestras tal como fueron recibidas fueron $0.041 \mathrm{~mm}$ (Cu_barra), $0.013 \mathrm{~mm}$ (Cu_placa), $0.016 \mathrm{~mm}(\mathrm{CuBe}), 0.07 \mathrm{~mm}$ (A1 CuAlBe) y $0.25 \mathrm{~mm}$ (A2 CuAlBe). Se obtuvieron diferentes tamaños de grano mediante tratamientos térmicos a $840^{\circ} \mathrm{C}$ ejecutados durante distintos tiempos finalizando con templado en agua a temperatura ambiente. La microestructura de las muestras de aleaciones base cobre a temperatura ambiente corresponde a una solución sólida con estructura fcc $(\mathrm{CuBe})$ y fase $\beta \mathrm{DO}_{3}(\mathrm{CuAlBe})$. Para observaciones microscópicas de las muestras en base $\mathrm{Cu}$ se trató previamente las muestras mediante electropulido en una solución saturada de trióxido de cromo y ácido fosfórico a aproximadamente $4 \mathrm{~V}$. Posteriormente se sumergieron por algunos segundos en una solución de cloruro férrico. Las micrografías fueron obtenidas usando un microscopio Reichert N384-180 con un adquisidor de imágenes Sony. Los tamaños de grano fueron estimados a partir de las imágenes obtenidas por microscopía óptica, mediante el método de análisis de área. Utilizando el software Image Tool 3.0 se midió el área de cada grano y se obtuvo un diámetro equivalente $(d)$ suponiendo que cada grano tiene forma circular. La configuración experimental para mediciones IET fue implementada específicamente en nuestro laboratorio. Sobre dos soportes de geometría diédrica de ángulo agudo se sostiene la muestra. Los soportes están ubicados a una distancia de 0.224 de la longitud desde los bordes de la muestra. La vibración de la barra se produce por el impacto de una bolilla plástica en el centro de la barra, vibración que fue detectada mediante un micrófono electrete unido a un circuito amplificador que permitió almacenar las señales en una computadora personal. Las señales fueron monitoreadas usando un osciloscopio Tektronix TBS 1022.

De cada una de las evoluciones temporales que surgen de las oscilaciones de las barras se realizó un análisis de Transformada Rápida de Fourier obteniendo los espectros de frecuencias para cada una de ellas. 
Se obtuvo en cada caso la frecuencia fundamental de oscilación de la barra.

Para la frecuencia fundamental (f), el módulo $E$ de una barra de sección rectangular está dado por [12]:

$$
E=0.9465\left(\frac{m f^{2}}{w}\right)\left(\frac{L^{3}}{t^{3}}\right) T
$$

, donde $m$ es la masa de la muestra, $w, t$ y $L$ son el ancho, espesor y longitud de la muestra, respectivamente, y $T$ es un factor de corrección.

Para el caso de vibración en modo fundamental en flexión de barras de sección circular, $E$ fue obtenido usando [12]:

$$
E=1.261886 \frac{4 m L^{3} f^{2}}{\pi D^{4}} T_{2}
$$

, donde $D$ es el diámetro de la barra, $T_{2}$ es un factor de corrección calculado usando la aproximación dada en [3], y $m$ y $L$ se definen de la misma manera que en la ecuación (1).

Para realizar las mediciones IET a distintas temperaturas se agregó al dispositivo un sistema de calentamiento de diseño propio. Se utilizaron dos dispositivos experimentales distintos: para barras cuadradas se construyó un horno pequeño que encierre la muestra, mientras que para barras circulares las muestras se sujetaron a la mitad de su longitud, se colocó la misma dentro de un horno eléctrico y se determinó la frecuencia de vibración en modo longitudinal excitando mediante un impulso en uno de los extremos. En este caso la expresión que permite calcular $E$ a partir de la frecuencia es [12]:

$$
E=\frac{1}{K_{n}} \frac{4 m}{\pi D^{2} L}\left(\frac{2 L}{n}\right)^{2} f^{2}
$$

En la ecuación (3), $n$ es el modo de vibración y $K_{n}$ un factor de corrección, que fue estimado siguiendo la aproximación descripta en [3], ( $m, L, f, D$ han sido definidas en el texto precedente). La ventaja de este último método es que permite un mejor control de la muestra y evita desplazamientos de la misma luego del impacto. Además, se pueden utilizar hornos comúnmente usados para realizar tratamientos térmicos y alcanzar mayores temperaturas. Un inconveniente que se presenta es la dificultad que se observa para acercar el micrófono de detección a la muestra debido al riesgo de inutilizarlo por efecto de la alta temperatura. Para resolver esa situación las ondas sonoras producidas se canalizaron por un conducto tubular de vidrio montado con un extremo junto a la muestra y el otro fuera del horno, donde se ubicó el micrófono. Se verificó que los resultados de $E$ obtenidos a temperatura ambiente usando este método coinciden con los obtenidos usando la frecuencia de vibración en flexión sin usar la canalización.

Se utilizó un controlador de temperatura electrónico para ejecutar un calentamiento controlado de las muestras. La temperatura se midió con una termocupla tipo K colocada en cerrada proximidad a las muestras, sin establecer contacto directo. El aumento de la temperatura se ejecutó en forma escalonada, permitiendo, en cada paso, que la temperatura se estabilice manteniendo para ello el sistema en espera durante algunos minutos.

\section{RESULTADOS}

En la Figura 1 se presentan micrografías representativas de muestras de $\mathrm{Cu}$ placa, $\mathrm{CuBe}$ y $\mathrm{CuAlBe}$ antes y después de ser sometidas a tratamientos térmicos (TT) que son especificados en la misma Figura. 


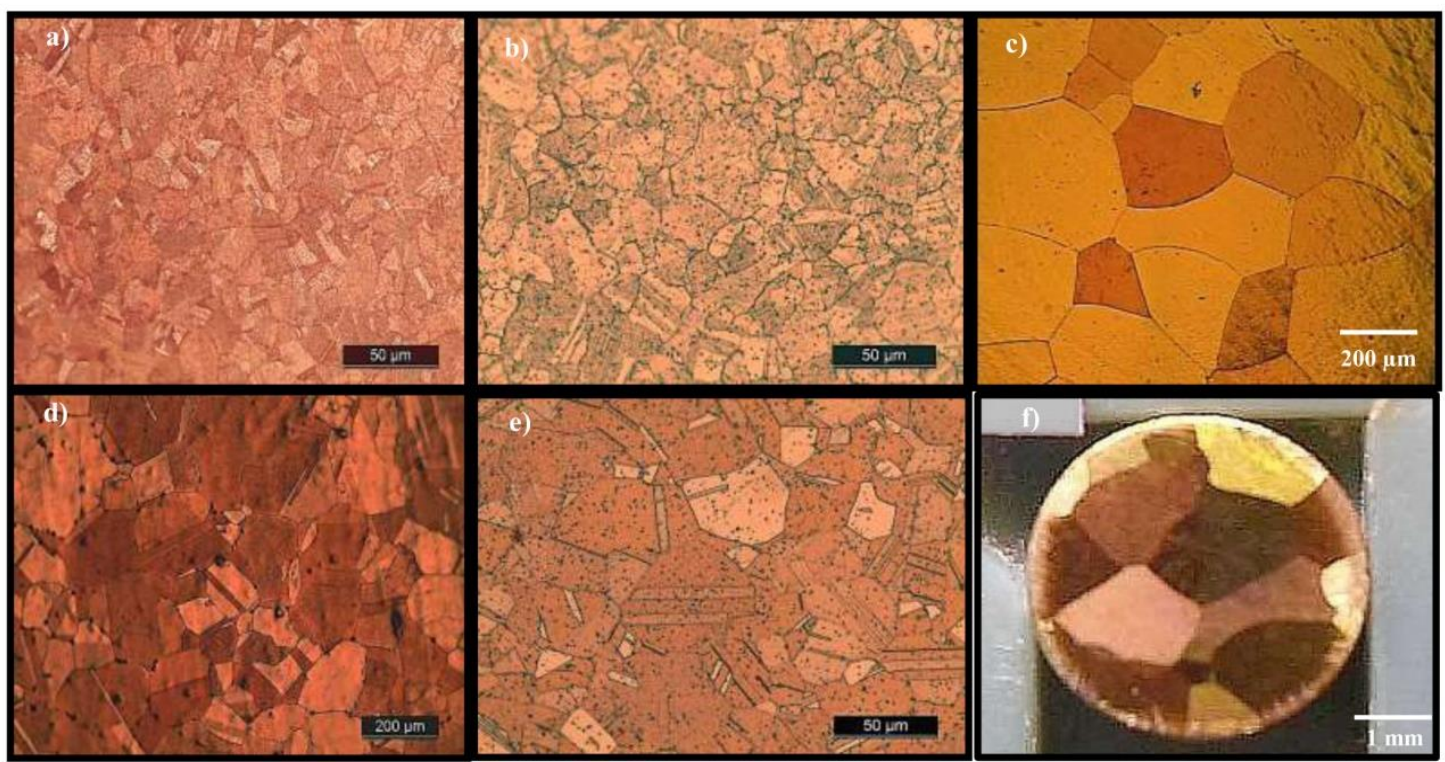

Figura 1: Micrografías correspondientes a a) cobre, b) $\mathrm{CuBe}$ y c) CuAlBe antes del tratamiento térmico; d) cobre y e) $\mathrm{CuBe}$ luego de $30 \mathrm{~min}$ a $840^{\circ} \mathrm{C}$ y temple; y f) CuAlBe luego de 15 min a $840^{\circ} \mathrm{C}$ y temple.

En la Figura 2 se presentan los resultados obtenidos midiendo el diámetro de grano $(d)$ en función del tiempo $(t)$ de $\mathrm{TT}$ a $840^{\circ} \mathrm{C}$. Se observa que las muestras de $\mathrm{Cu}$ placa y $\mathrm{CuBe}$ muestran un incremento del tamaño de grano en función del incremento del tiempo de TT. Sin embargo, no se observa incremento del tamaño de grano en función de $t$ en la muestra de Cu conformada como barra cilíndrica. A modo de comparación se presentan los resultados de crecimiento de grano de la aleación CuAlBe reportada por los autores en [3], que se corresponde con la aleación A2 de este trabajo. La aleación CuAlBe y el Cu laminado muestran un incremento del tamaño de grano en función de $t$ mucho mayor que el observado en $\mathrm{CuBe}$. Es conocido que los tratamientos termomecánicos ejecutados durante la fabricación de las aleaciones también influyen sobre la posterior respuesta en lo que respecta a crecimiento de grano y sobre el tamaño de grano inicial medido. Es por ello que la muestra de $\mathrm{Cu}$ en forma de placa con una mayor deformación previa (muestra laminada) exhibe un mayor crecimiento de grano que la muestra provista con forma de barras cilíndricas, la cual no presenta un crecimiento de grano significativo.

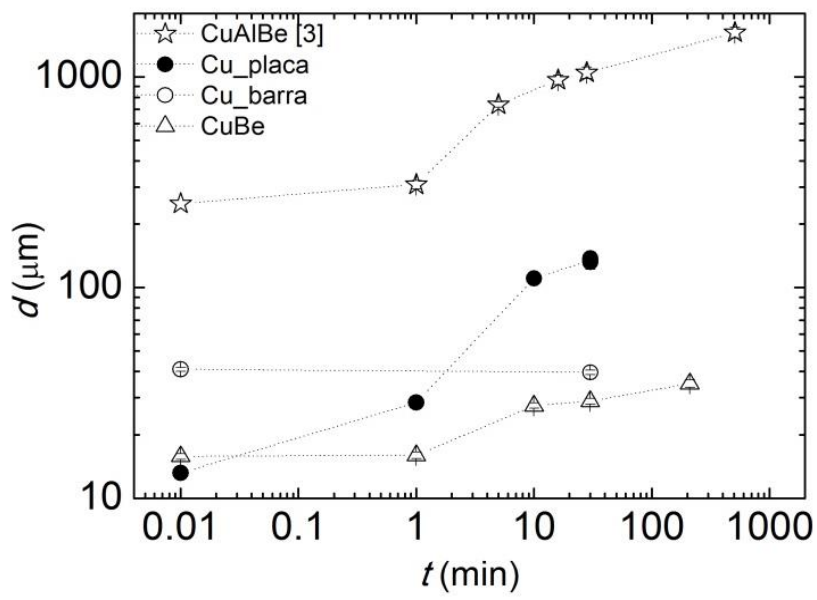

Figura 2: Diámetro del grano $d$ en función del tiempo a $840{ }^{\circ} \mathrm{C}$. Al valor de $d$ sin tratamiento térmico se ha asignado arbitrariamente el valor $0.01 \mathrm{~min}$

Se observó que la distribución de tamaño de grano (diámetro $d$ ) no se modifica con el aumento del tamaño promedio $\left(d_{\text {prom }}\right)$. Efectivamente, puede observarse en la Figura 3 en un gráfico de frecuencia estadística relativa en función de $d / d_{\text {prom }}$ que todas las muestras estudiadas antes y luego de sometidas a tratamiento térmico tienen una distribución similar. 


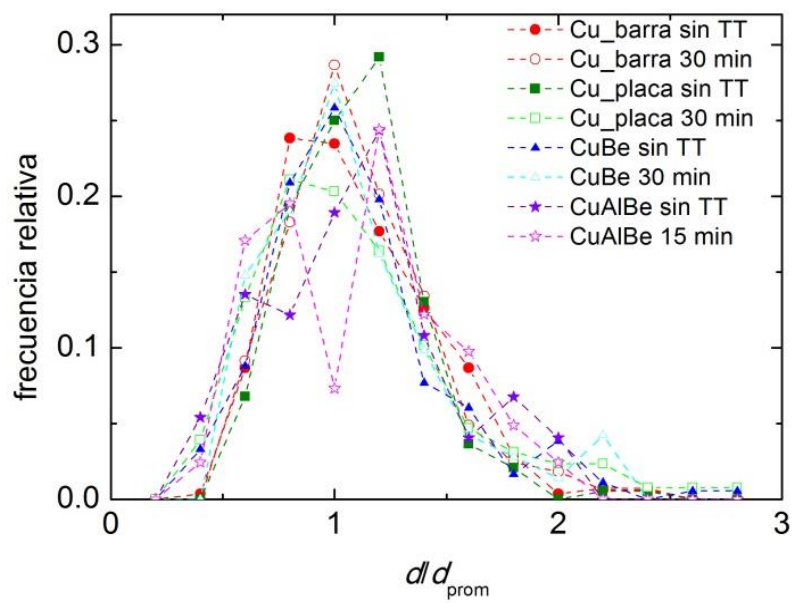

Figura 3: Distribución de tamaño de grano normalizado al tamaño promedio $\left(d / d_{\text {prom }}\right)$ para las distintas muestras estudiadas sin tratamiento térmico ( $\sin \mathrm{TT})$ y con 15 ó $30 \min$ a $840^{\circ} \mathrm{C}$.

Se determinó el módulo $E$ en muestras con distintos tamaños de grano, mostrándose los valores en la Tabla 1. En la Figura 4 se presentan los resultados en función de $d$ para $\mathrm{CuBe}$ y $\mathrm{Cu}$. Se observa que en la muestra de $\mathrm{Cu}$ laminado el módulo $E$ sólo presenta una leve variación a pesar de que el tamaño de grano creció casi 10 veces su tamaño original. La muestra de $\mathrm{CuBe}$ presenta una disminución importante del módulo $E$ al aumentar $d$. La dependencia del módulo $E$ con el tamaño de grano para $\mathrm{CuAlBe}$ fue reportada en [3], observando que esta aleación, al tener un mayor crecimiento del tamaño de grano llega a valores de $E$ de $43 \%$ del valor inicial, mientras que para $\mathrm{CuBe}$ sólo se llega a un $95 \%$ del valor inicial. Las barras de cobre de forma cilíndrica también fueron medidas mediante IET, encontrando un valor de $117.7 \mathrm{GPa}$. Este valor es poco mayor que la barra de $\mathrm{Cu}$ laminada con un tamaño de grano similar.

Tabla 1: Módulo E en función de d para $\mathrm{Cu}$ laminado, $\mathrm{Cu}$ en barra y $\mathrm{CuBe}$, para diferente tamaño de grano (diámetro d), ver texto.

\begin{tabular}{l|l|l|l|l|l}
\hline \multicolumn{2}{l}{ COBRE LAMINADO } & \multicolumn{2}{l|}{ COBRE EN BARRA } & \multicolumn{2}{l}{ ALEACION COBRE BERILIO } \\
\hline$d(\mu \mathrm{m})$ & $E(\mathrm{GPa})$ & $d(\mu \mathrm{m})$ & $E(\mathrm{GPa})$ & $d(\mu \mathrm{m})$ & $E(\mathrm{GPa})$ \\
\hline 13.2 & $116.6 \pm 3.9$ & 40.9 & $118.7 \pm 1.4$ & 15.8 & $126.1 \pm 4.8$ \\
\hline 28.5 & $116.7 \pm 3.9$ & 39.6 & $118.9 \pm 1.5$ & 16.0 & $124.4 \pm 4.5$ \\
\hline 110.5 & $116.7 \pm 3.9$ & & & 27.5 & $121.1 \pm 4.6$ \\
\hline 134.6 & $113.8 \pm 3.8$ & & & 28.9 & $120.5 \pm 4.6$ \\
\hline & & & & 35.0 & $120.4 \pm 4.4$ \\
\hline
\end{tabular}




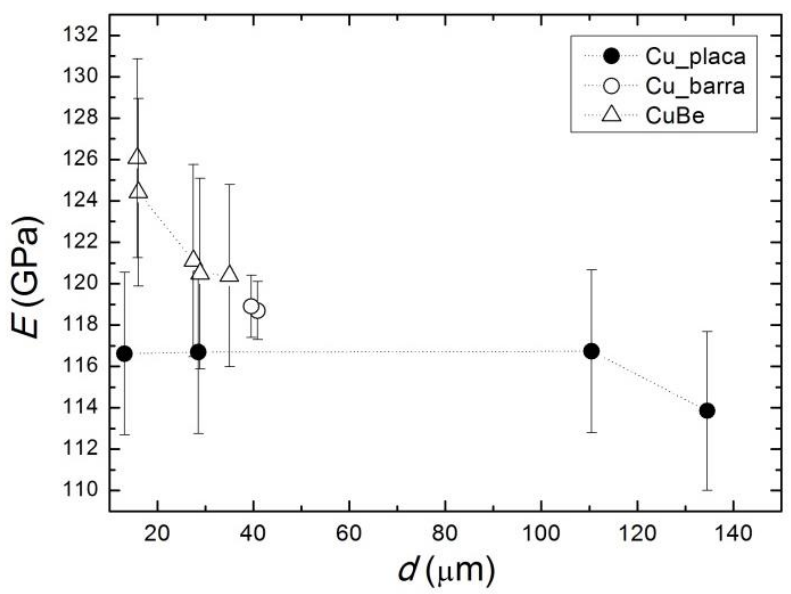

Figura 4: Módulo $E$ en función de $d$ para $\mathrm{Cu}$ laminado $\left(\mathrm{Cu} \_\right.$placa $), \mathrm{Cu}$ en barra $\left(\mathrm{Cu} \_\right.$barra $)$y $\mathrm{CuBe}$.

Con el fin de analizar el módulo $E$ en función del tamaño de grano se utilizó la ecuación 4, propuesta en Ref. [13]:

$$
E=E_{0}-b \cdot \log (S)
$$

donde $S$ el área del grano, y $E_{0}$ y $b$ constantes. El área fue estimada como $\pi(d / 2)^{2}$. En la Figura 5 se presentan los valores de $E$ en función de $S$ para las muestras $\mathrm{CuBe}$ y $\mathrm{CuAlBe}$ y los ajustes efectuados usando la ecuación 4. Los valores obtenidos para las constantes $E_{0}$ y $b$ resultan: $143.20 \mathrm{GPa}$ y 7.86 para CuBe, y $150.35 \mathrm{GPa}$ y 16.65 para $\mathrm{CuAlBe}$, respectivamente. La constante $E_{0}$ podría ser analizada como el valor para el caso de un tamaño de grano igual a $1 \mu \mathrm{m}^{2}$, lo cual puede ser considerado como un grano extremadamente pequeño. La constante $b$, por su parte presenta un valor mucho mayor para el $\mathrm{CuAlBe}$, indicando una mayor dependencia de $E$ con el tamaño de grano para esta aleación.
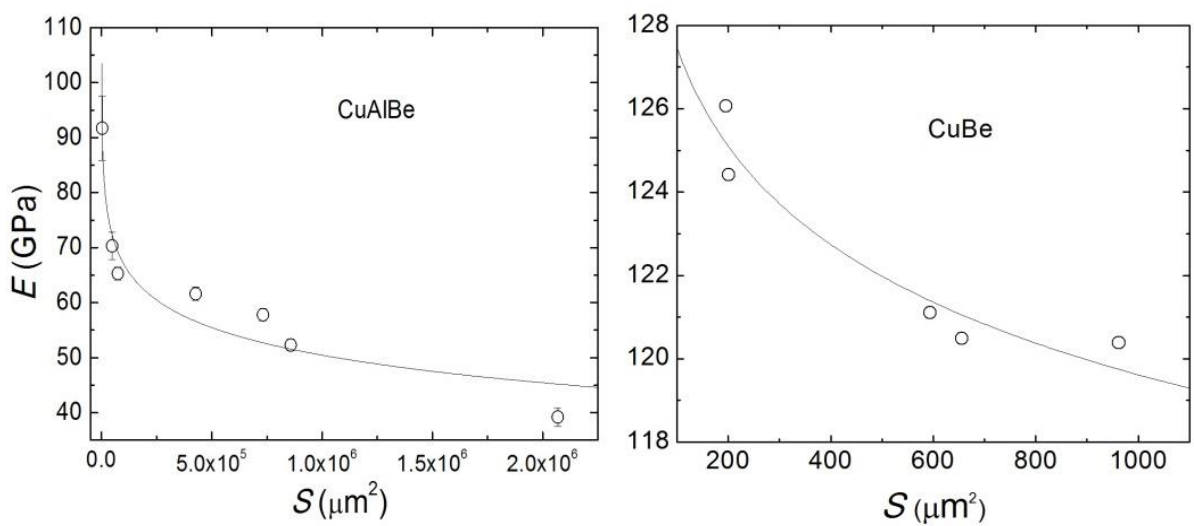

Figura 5: Módulo $E$ en función del área $S$ y ajuste mediante la ecuación 4.

Para estimar los valores de $E$ en policristales se utilizan generalmente los modelos de Voigt y de Reuss, que definen valores máximo y mínimo para el valor de $E$ [14]. El modelo de Voigt asume una deformación uniforme mientras que el modelo de Reuss asume un esfuerzo uniforme. El modelo de Hill es un promedio entre los modelos de Voigt y Reuss [15]. En la Figura 6 se presentan los resultados medidos experimentalmente de $E$, junto con el resultado arrojado por el cálculo según los modelos mencionados. Las constantes elásticas fueron obtenidas de [16] para CuAlBe, [17] para $\mathrm{Cu}$ y aluminio, y [18] para CuBe. Para $\mathrm{CuBe}$ y $\mathrm{CuAlBe}$ se ha representado el valor correspondiente al menor y al mayor tamaño de grano obtenido en este trabajo. Se puede observar que para el $\mathrm{Cu}$ los resultados experimentales resultan por debajo de las estimaciones mediante el modelo de Hill. Para el caso de $\mathrm{CuBe}$ los resultados experimentales resultan cercanos a los estimado mediante el modelo de Hill, ya sea para el mayor o el menor tamaño de grano. Sin embargo, para 
$\mathrm{CuAlBe}$ el resultado experimental es marcadamente inferior al estimado para el tamaño de grano menor, para el tamaño de grano mayor resulta fuera de los límites esperados. Esta discrepancia podría ser analizada como una textura de este material. A modo de comparación se ha añadido a la Figura el resultado correspondiente a Al puro comercial, en el cual se observa una cerrada coincidencia entre el resultado experimental y estimado. Es importante notar que los resultados de $E_{0}$ obtenidos mediante el ajuste de $E$ en función del área del grano para $\mathrm{CuBe}$ y $\mathrm{CuAlBe}$, los cuales también se incluyen en la Figura 6, son similares a los estimados mediante el modelo de Voigt.

Como un comentario adicional se menciona que Shirakawa y Numakura [13] observaron que en aleaciones $\mathrm{NiCu}$ los valores de $E$ para los mayores tamaños de grano tienden al valor de $E$ para un monocristal en la orientación [100], la cual es la que permite alcanzar el valor mínimo de $E$. Si se extrapola la curva ajustada para $\mathrm{CuAlBe}$ se encuentra que el valor mínimo, que en este caso sería $\approx 21 \mathrm{GPa}$, se alcanzaría para un valor de $d$ de aproximadamente $\approx 8800 \mu \mathrm{m}$, aproximadamente 5.4 veces superior al valor de $d$ más alto observado en este trabajo. Sin embargo, para CuBe el valor en la dirección [100] sería $\approx 50 \mathrm{GPa}$, y para llegar a ese valor sería necesario un $d$ de $\approx 874000 \mu \mathrm{m}, 25000$ veces el mayor valor de $d$ alcanzado en este trabajo. Este hecho señalaría una textura morfológica de la muestra CuAlBe.

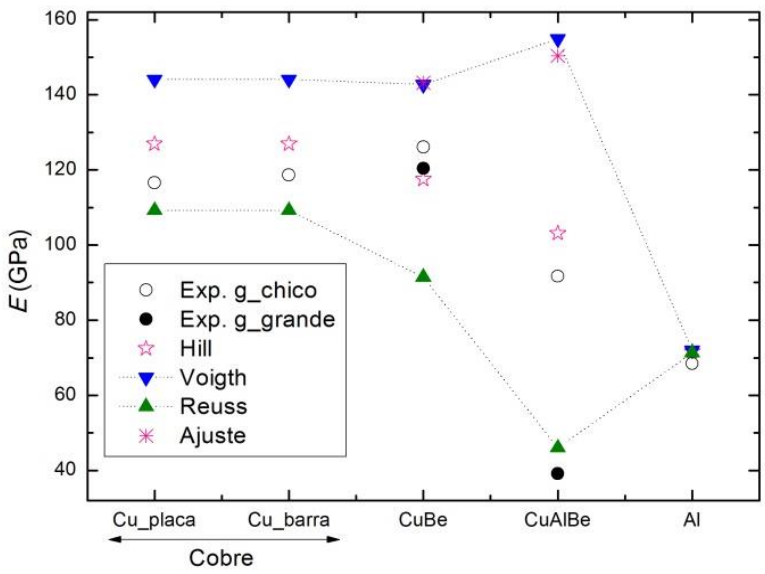

Figura 6: Módulo $E$ experimental para muestra de grano chico (menor tamaño de grano obtenido en este trabajo) y muestra de grano grande (mayor tamaño de grano obtenido en este trabajo), y calculado mediante los modelos de Voigt, Reuss y Hill para las distintas muestras estudiadas. Se agregaron los valores de $E_{0}$ obtenidos del ajuste de $E$ en función de $S$ (ver texto).

\subsection{Medición del módulo $E$ a diferentes temperaturas}

Con la intención de explorar los alcances de la técnica IET en el estudio de materiales a diferentes temperaturas y obtener información de la variación que se produce en el módulo $E$, se realizaron mediciones a distintas temperaturas. En la Figura 7 se presentan los valores de $E$ calculados a partir de la frecuencia de resonancia, para muestras de aluminio, $\mathrm{CuAlBe}$ y $\mathrm{CuBe}$ en un barrido desde temperatura ambiente hasta temperaturas entre 70 y $170^{\circ} \mathrm{C}$, dependiendo del material. Se observa que existe una disminución del módulo $E$ en función del aumento de la temperatura de manera aproximadamente lineal.

Los resultados obtenidos se ajustaron mediante una función lineal de la forma propuesta en la ecuación (5), siendo $\alpha$ y $\beta$ parámetros de ajuste, pendiente y ordenada al origen, respectivamente.

$$
E=\beta-\alpha \cdot T
$$

En la Figura 7 se presentan los valores arrojados por el ajuste de las distintas muestras. 


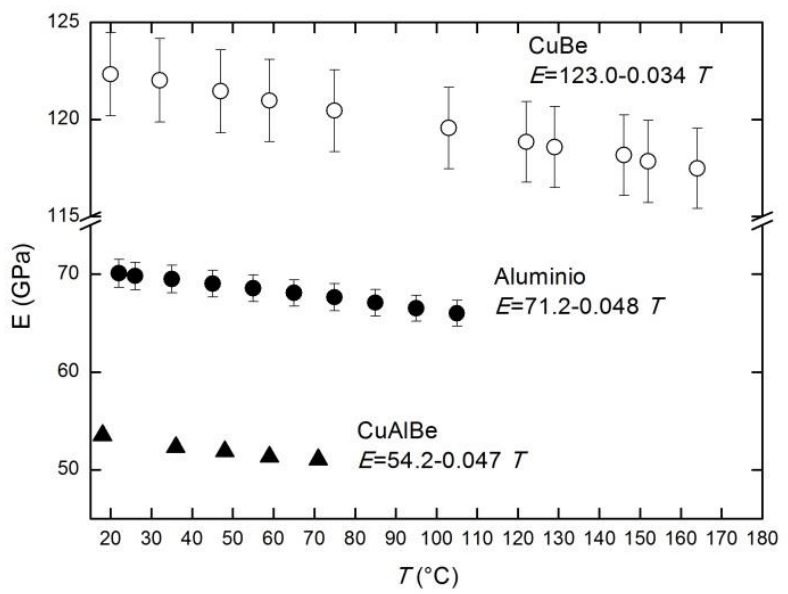

Figura 7: Módulo $E$ en función de la temperatura para $\mathrm{CuBe}, \mathrm{CuAlBe}$ y aluminio.

Para analizar la variación de $E$ con la temperatura, se utilizaron dos modelos distintos dados por las ecuaciones 6 y 7, respectivamente, propuestas en [19], vinculando la dependencia de la constante elástica $c_{11}$ con la temperatura:

$$
\begin{aligned}
& \mathrm{c}_{11}=C_{1}-\frac{C_{2} \cdot T^{2}}{\left(T+C_{3}\right)} \\
& \mathrm{c}_{11}=C_{0}-\frac{\mathrm{s}}{e^{\frac{T_{0}}{T}}-1}
\end{aligned}
$$

De manera similar se calcularon las constantes $\mathrm{c}_{12} \mathrm{y} \mathrm{c}_{44}$, en función de la temperatura mediante expresiones análogas a (6) para un modelo o (7) para otro, pero tomando en consideración que los parámetros $C_{1}$, $C_{2}, C_{3}, C_{0}, s$ y $T_{0}$ son diferentes para cada una de las constantes elásticas. Los mismos fueron extraídos de [19] para $\mathrm{Al}$ y $\mathrm{Cu}$. Con estos valores se calculó el valor de $E$ para un policristal usando el modelo de Hill para cada temperatura, y de los mismos se determinó la pendiente de una función lineal ajustada representando $E$ en función de $T$. El valor para $\mathrm{Al}$ así calculado resultó 0.042 usando la ecuación (6) y 0.038 usando la ecuación (7). Para el $\mathrm{CuBe}$ se utilizaron los parámetros correspondientes al $\mathrm{Cu}$, obteniendo una pendiente de 0.052 usando la ecuación (6) y 0.047 usando la ecuación (7). Para el CuAlBe se consideró la dependencia lineal con la temperatura dada por Rios Jara et al. [16] obtenida mediante mediciones de ultrasonido y de las mismas se calcularon $\mathrm{c}_{11}, \mathrm{c}_{12}$ y $\mathrm{c}_{44}$ a distintas temperaturas. Mediante el modelo de Hill se calculó $E$ a diferentes temperaturas, y en este caso la dependencia con la temperatura de $E$ mostró una pendiente igual a 0.026 , bastante inferior a la obtenida experimentalmente.

La diferencia entre el valor experimental y el esperado en el $\mathrm{CuBe}$ se puede asociar a una diferente dependencia de las constantes elásticas con la temperatura respecto al $\mathrm{Cu}$. Sin embargo, en el caso de $\mathrm{CuAl-}$ $\mathrm{Be}$, la diferencia sería atribuible a una posible textura de la muestra.

\section{CONCLUSIONES}

En este trabajo se estudió la influencia del tamaño de grano sobre el módulo de Young en cobre y aleaciones base cobre, utilizando la técnica IET. El tamaño de grano fue modificado a partir de tratamientos térmicos, encontrando un crecimiento mayor para la aleación CuAlBe y para el cobre en forma de placa. La aleación de CuBe mostró una menor variación del tamaño de grano, mientras que el cobre en forma de barra prácticamente no modificó su valor. Se encontró que el módulo de Young varía fuertemente en función del tamaño de grano en $\mathrm{CuAlBe}$, en menor medida en $\mathrm{CuBe}$ y no presenta dependencia en el cobre. Los resultados fueron analizados en términos de los modelos de Voigt, Reuss y Hill. 
Se determinó el módulo de Young a diferentes temperaturas y se aplicaron modelos de la literatura para analizar la variación del módulo en policristales, en función de la variación de las constantes elásticas con la temperatura. En la aleación $\mathrm{CuAlBe}$ se presentaron las mayores discrepancias con los valores esperados de los cálculos, lo que podría ser atribuible a la presencia de textura en las muestras.

La técnica IET mostró ser una variante simple y de bajo costo para determinar variaciones del módulo de Young ante variaciones microestructurales, en este caso tamaño de grano, o de la temperatura.

\section{AGRADECIMIENTOS}

Los autores agradecen al Consejo Nacional de Investigaciones Científicas y Técnicas (CONICET), Comisión de Investigaciones Científicas de la Provincia de Buenos Aires (CICPBA), programa CIC para proyectos de interés científico y tecnológico 2013, Res. 813/13 y Res 1707/15, SECAT (UNCPBA) and ANPCyTFONCyT, PICT 2013-0686, and PICT 2012-0868, Argentina.

\section{BIBLIOGRAFÍA}

[1] RADOVIC, M., LARA-CURZIO, E., RIESTER, L., “Comparison of different experimental techniques for determination of elastic properties of solids”, Mater Sci Eng A, v. 368, pp. 56-70, Marzo 2004.

[2] HERITAGE, K., FRISBY C., WOLFENDEN, A., "Impulse excitation technique for dynamic flexural measurements at moderate temperature", Review of Scientific Instruments, v. 59, pp. 973-974, Junio 1988.

[3] MONTECinOS, S., TOGNANA, S., SAlgueIRO, W., "Determination of the Young's modulus in $\mathrm{CuAlBe}$ shape memory alloys with different microstructures by impulse excitation technique", Mater Sci Eng A, v. 676, pp. 121-127, Agosto 2016.

[4] DUAN, R-G., ROEBBEN, G., VLEUGELS, J., et al., "Optimization of microstructure and properties of in situ formed $\beta$-O-sialon-TiN composite”, Mater Sci Eng A, v. 427, pp. 195-202, Julio 2006.

[5] CASCIATI, F., FARAVELLI, L., "Experimental characterization of a Cu-based shape memory alloy toward its exploitation in passive control devices", J Phys IV, v. 115, pp. 299-306, Junio 2004.

[6] SAINT-SULPICE, L., CHIRANI, S.A., CALLOCH, S., "Super-elastic behavior of shape memory alloys under proportional cyclic loadings", Mater Sci Eng A, v. 481-482, pp. 174-7, Mayo 2008.

[7] BOUVET, C., CALLOCH, S., LEXCELLENT, C., "Mechanical behavior of a Cu-Al-Be shape memory alloy under multiaxial proportional and nonproportional loadings", Trans ASME, v. 124, pp. 112-24, Marzo 2002.

[8] MONTECINOS, S., CUNIBERTI, A., SIMISON, S., "Instrumented indentation of transforming and notransforming phases in Cu-Al-Be shape-memory alloys", Intermetallics, v. 28, pp. 58-64, Septiembre 2012.

[9] YAGMUR, L., "Effect of microstructure on internal friction and Young's modulus of aged Cu-Be alloy", Mater Sci Eng A, v. 523, pp. 65-69, Octubre 2009.

[10] MONTECINOS, S., CUNIBERTI, A., ROMERO, R., "Effect of grain size on the stress-temperature relationship in a $\beta$ CuAlBe shape memory alloy”, Intermetallics, v. 19, pp. 35-38, Enero 2011.

[11] MIKI, M., TENMA, A., ISHIKAWA, S., OGINO, Y., "Influence of grain size and quenching rate on the age-hardening behavior of Cu-2\%Be alloy”, Mater. Trans. JIM, v. 39, № 4, pp. 445-462, 1998.

[12] SPINNER, S., TEFFT, W., "A method for determining mechanical resonance frequencies and for calculating moduli from these frequencies”, Proceedings ASTM, v. 61, pp. 1221-1238, 1961.

[13] SHIRAKAWA, Y., NUMAKURA, K., "On Young's Modulus and Grain Size in Nickel-Copper Alloys". In: The 904th report of the Research Institute for Iron, Steel and Other Metals, Published in Japanese in Nippon Kinzoku Gakkai-shi (J .Japan Inst. Metals), v. 19, 1955.

[14] MURA, T., Micromechanics of Defects in Solids, 2 ed. Netherlands, Martinus Nijhoff Publishers, 1987.

[15] HILL, R., "The elastic behaviour of a crystalline aggregate", Proc Phys Soc A, v. 65, pp. 349-354, 1952.

[16] RIOS-JARA, D., BELKAHLA, S., CANALES, A., et al., "Elastic constants measurements of $\beta$ Cu-AlBe alloy”, Scr Metall Mater, v. 25, pp. 1351-1355, Marzo 1991.

[17] COURTNEY, T.H., Mechanical Behavior of Materials, 2 ed., Illinois, Waveland Press Inc, 2000.

[18] KOO, Y.M., COHEN, J.B., "Estimation of monocrystal elastic constants for an alloy composed of a cubic solvent and a hexagonal solute”, Mater Sci Eng, v. 91, pp. L5-L8, Julio 1987. 
[19] VARSHNI, Y.P., “Temperature Dependence of the Elastic Constants", Physical Review B, v. 2, n. 10, pp. 3952-3958, Nov. 1970. 